\title{
Procura de Serviço Médico por Mulheres com Incontinência Urinária
}

\author{
The Search of Medical Care by Women with Urinary Incontinence
}

Telma Guarisi, Aarão Mendes Pinto-Neto, Maria José Osis, Adriana Orcesi Pedro

Lucia Helena Simões Costa-Paiva, Aníbal Faúndes

\begin{abstract}
RESUMO
Objetivo: avaliar a porcentagem de mulheres de 45 a 60 anos com queixa de perda urinária que solicitam tratamento médico, possiveis fatores associados à decisão de consultar e motivos para não fazê-lo.

Métodos: realizou-se análise secundária de dados de um inquérito domiciliar sobre o climatério e a menopausa entre mulheres. Por meio de estudo descritivo e exploratório de corte transversal de base populacional, foram selecionadas, por processo de amostragem, 456 mulheres, na faixa etária de 45-60 anos de idade. Avaliaram-se a idade à menopausa e seus fatores associados, prevalência de sintomas climatéricos, uso de serviço médico, autopercepção do estado de saúde e características socioculturais, demográficas e econômicas. Exploraram-se a queixa de perda urinária aos esforços e a procura por atendimento médico em virtude dessa queixa, bem como os fatores que interferiram nessa procura. Os dados foram coletados por meio de entrevistas domiciliares, com questionários estruturados e pré-testados, fornecidos pela Fundação Internacional de Saúde/Sociedade Internacional de Menopausa e pela Sociedade Norte-Americana de Menopausa, adaptados pelos autores. A análise dos dados foi realizada pelo teste exato de Fisher.

Resultados: das pacientes entrevistadas, 35\% queixaram-se de perda urinária aos esforços, sempre ou às vezes; no entanto, somente 59\% das mulheres com queixa procuraram ajuda médica. O principal motivo apontado como responsável pela não-procura foi o fato de acharem que os sintomas não mereciam atenção médica.

Conclusão: grande parte das mulheres com incontinência urinária não relata a queixa ao médico se não forem objetivamente questionadas.
\end{abstract}

PALAVRAS-CHAVE: Incontinência urinária. Menopausa. Atenção médica.

\section{Introdução}

A incontinência urinária ocorre entre 30 e $60 \%$ de todas as mulheres durante o período de climatério e na menopausa, tendo importância, portanto, pela sua alta incidência e também porque afeta negativamente a qualidade de vida dessas mulheres.

Departamento de Tocoginecologia da Faculdade de Ciências Médicas da Universidade Estadual de Campinas.

Correspondência:

Aarão Mendes Pinto Neto

Rua Alexander Fleming, 101 - Cidade Universitária "Zeferino Vaz"

13.083-970 - Campinas - SP

Fone (19) 3788-9402 / Fax: (19) 3289-5935

Apoio: Fundação de Amparo à Pesquisa do Estado de São Paulo (FAPESP 96/10341-2) e Fundo de Apoio ao Ensino e à Pesquisa - Faculdade de Ciências Médicas - Universidade Estadual de Campinas (FAEP 008/98).
Diferenças na prevalência têm sido descritas em diferentes populações ${ }^{1}$. No Brasil são poucos os estudos sobre prevalência de incontinência urinária, e não encontramos na literatura nacional nenhuma publicação que tenha avaliado esta prevalência em inquéritos de base populacional. Por isto, não encontramos informação sobre a proporção de mulheres com incontinência urinária que consultam médico por este problema ou estudos que tentaram identificar possiveis fatores associados à procura de ajuda para corrigir a incontinência.

Sabe-se, entretanto, que uma proporção elevada das mulheres com este sintoma não consulta espontaneamente ${ }^{2}$, pelo que parece evidente que o médico deve questionar a respeito da perda involuntária de urina. Butler et al. $^{3}$ afirmam que uma história clínica cuidadosa pode revelar 80 a 90\% dos diagnósticos. 
Estes questionamentos motivaram o presente estudo, que investigou a prevalência de incontinência urinária de esforço, bem como a porcentagem de mulheres que procurou atendimento médico por esta queixa e o motivo de não consultar, entre mulheres de 45 a 60 anos, por meio de inquérito populacional.

\section{Pacientes e Métodos}

Trata-se de um estudo descritivo e exploratório de corte transversal, tipo inquérito populacional domiciliar. Para o cálculo do tamanho amostral considerou-se uma proporção populacional de mulheres com sintomatologia geral do climatério de $60 \%$, entre eles a incontinência urinária ${ }^{4}$, com uma diferença máxima desejada entre a proporção amostral e populacional de $5 \%$, e um erro tipo I (alfa) de 0,05 . A populaçãoalvo considerada foi a feminina de Campinas, na faixa etária entre 45-60 anos, no ano de 1997. Obteve-se este dado por uma projeção, por meio de regressão linear da população, baseada no número de mulheres entre 45 a 60 anos residentes no município de Campinas no Censo Demográfico de 1990 (IBGE, 1994)5 com projeção estimada para o ano de 1997, sendo este total de 79.727. Tal procedimento foi realizado pelo Laboratório Aplicado de Epidemiologia do Departamento de Medicina Social da Universidade Estadual de Campinas (UNICAMP), utilizando-se a regressão linear da população, baseando-se em método descrito por Laurenti et al. ${ }^{6}$. Desta forma, o número mínimo calculado de entrevistas foi de 367 mas foi acrescido de $20 \%$, levando-se em consideração o número de prováveis usuárias de terapia de reposição hormonal, resultando em um total de 456 entrevistas. Este acréscimo se justifica porque mulheres em uso de anticoncepcionais hormonais ou em terapia de reposição hormonal seriam excluídas da análise da prevalência de sintomas climatéricos, visto que estas medicações poderiam mascará-los. A seleção das mulheres foi aleatória.

Um questionário estruturado e pré-testado foi utilizado para obter as informações, que foram relatadas pelas mulheres. O questionário foi elaborado a partir de outros dois questionários, adaptados pelos autores. O primeiro foi fornecido pela Sociedade Internacional de Menopausa e Fundação Internacional de Saúde, após ter sido aplicado em sete países do Sudeste Asiático em 19937. O segundo questionário, que havia sido aplicado nos Estados Unidos, foi fornecido pela Sociedade Norte-Americana de Menopausa ${ }^{8}$. O estrato socioeco- nômico foi determinado segundo critérios da Associação Brasileira de Anunciantes (ABA) e da Associação Brasileira dos Institutos de Pesquisa de Mercado (ABIPEME), classificado segundo a pontuação de Almeida e Wickerhauser ${ }^{9}$, em A (89 pontos ou mais), B (59-88 pontos), C (35-58 pontos), D (20-34 pontos) e E (0-19 pontos). O questionário utilizado para obtenção desta pontuação é padronizado pela ABA/ABIPEME, que considera o grau de escolaridade do chefe da família, número de eletrodomésticos, carros e presença de empregada doméstica. O estado menopausal foi definido utilizando o critério proposto por Jaszmann ${ }^{10}$ : prémenopausa: mulheres com ciclos menstruais regulares ou com padrão menstrual similar ao que elas tiveram durante a vida reprodutiva; perimenopausa: mulheres com ciclos menstruais nos últimos 12 meses, mas com mudança do padrão menstrual quando comparado aos padrões anteriores, e pós-menopausa: mulheres em que o último período menstrual ocorreu pelo menos 12 meses antes da entrevista.

Avaliou-se a presença ou não da queixa de perda urinária aos esforços, como tossir, rir ou levantar peso; avaliou-se ainda a procura de cuidado médico por esta queixa, bem como os fatores associados à necessidade ou não de procurar tratamento.

Os dados foram coletados por meio de entrevistas individuais em domicílio. A coleta de dados foi iniciada em outubro de 1997 e encerrada em janeiro de 1998, totalizando 73 dias de trabalho de campo.

O questionário foi estruturado para a realização de um amplo estudo de base populacional sobre o climatério. O mesmo foi organizado em cinco seções, que enfocaram perguntas relativas a: aspectos sociodemográficos, sexuais e reprodutivos (seção 1); atitudes em face da menopausa (seção 2); estado menopausal e sintomas (seção 3); conhecimento sobre a menopausa (seção 4) e classificação do status socioeconômico (seção 5).

A questão sobre incontinência urinária incluiu perda de urina quando a mulher tosse, ri ou carrega peso, segundo as categorias nunca, sempre e às vezes.

Para análise dos dados utilizou-se o teste exato de Fisher $(p<0,05)$ para verificar quais os fatores que interferiram na procura médica pelo problema.

\section{Resultados}

Do total de pacientes entrevistadas, 35\% referiram perda urinária aos esforços, sendo que 
$10,7 \%$ apresentavam o sintoma sempre e $24,3 \%$ às vezes. Das 160 mulheres com queixa, apenas pouco mais da metade $(58,8 \%)$ consultou um médico por este motivo. A proporção das mulheres que relataram consultar foi um pouco maior entre as mulheres que apresentavam perda urinária sempre do que em relação às que relataram o sintoma às vezes (Tabela 1).

Tabela 1 - Distribuição porcentual das pacientes com queixa de perda urinária, segundo a procura por consulta médica $(n=160)$.

\begin{tabular}{|c|c|c|c|c|c|c|}
\hline \multirow[t]{3}{*}{ Consulta médica } & \multicolumn{4}{|c|}{ Perda de urina aos esforços } & \multicolumn{2}{|c|}{ Total } \\
\hline & \multicolumn{2}{|c|}{ sempre } & \multicolumn{2}{|c|}{ às vezes } & \multirow[b]{2}{*}{$\mathbf{n}$} & \multirow[b]{2}{*}{$\%$} \\
\hline & $\mathrm{n}$ & $\%$ & $\mathrm{n}$ & $\%$ & & \\
\hline Sim & 31 & 63 & 63 & 57 & 94 & 58,8 \\
\hline Não & 18 & 37 & 48 & 43 & 66 & 41,2 \\
\hline Total & 49 & 100,0 & 111 & 100,0 & 160 & 100,0 \\
\hline
\end{tabular}

As mulheres relataram uma variedade de motivos que as levaram a não procurar atenção médica para este problema. A maior parte desses motivos sugere uma baixa valorização dos sintomas, como achar que eles não merecem atenção médica, não serem tão fortes ou não incomodarem, e falta de tempo para consultar. Quase 10\%, entretanto, relataram falta de liberdade de falar sobre estes sintomas com o médico (Tabela 2).

Tabela 2 - Porcentagem de pacientes que não consultaram médico, segundo o motivo $(n=66)$.

\begin{tabular}{lrr}
\hline Motivo & n & \%* $^{*}$ \\
\hline Os sintomas não mereciam atenção médica & 36 & 54 \\
Os sintomas não eram tão fortes ou não incomodavam & 9 & 14 \\
Não tinham tempo/tinham muito trabalho & 7 & 11 \\
Não sentiam liberdade de falar dos sintomas com o médico & 6 & 9 \\
Outros motivos & 11 & 16 \\
\hline *A porcentagem ultrapassou 100\% pelo fato de 3 pacientes apresentarem mais de um \\
motivo
\end{tabular}

Nenhum dos fatores estudados mostrou associação com a procura por atendimento médico para o problema da perda urinária. A proporção de mulheres que procurou médico foi significativamente maior entre aquelas da classe $C(p<0,05)$, sendo em torno de 25 pontos percentuais, menor tanto entre as mulheres das classes A e B como entre as que pertenciam às classes $\mathrm{D}$ e E, mostrando ausência de tendência segundo estrato social. Não houve diferenças na proporção de mulheres com sintomas que procurou atenção médica, segundo o grau de escolaridade, idade ou esta- do menopausal (Figura 1).
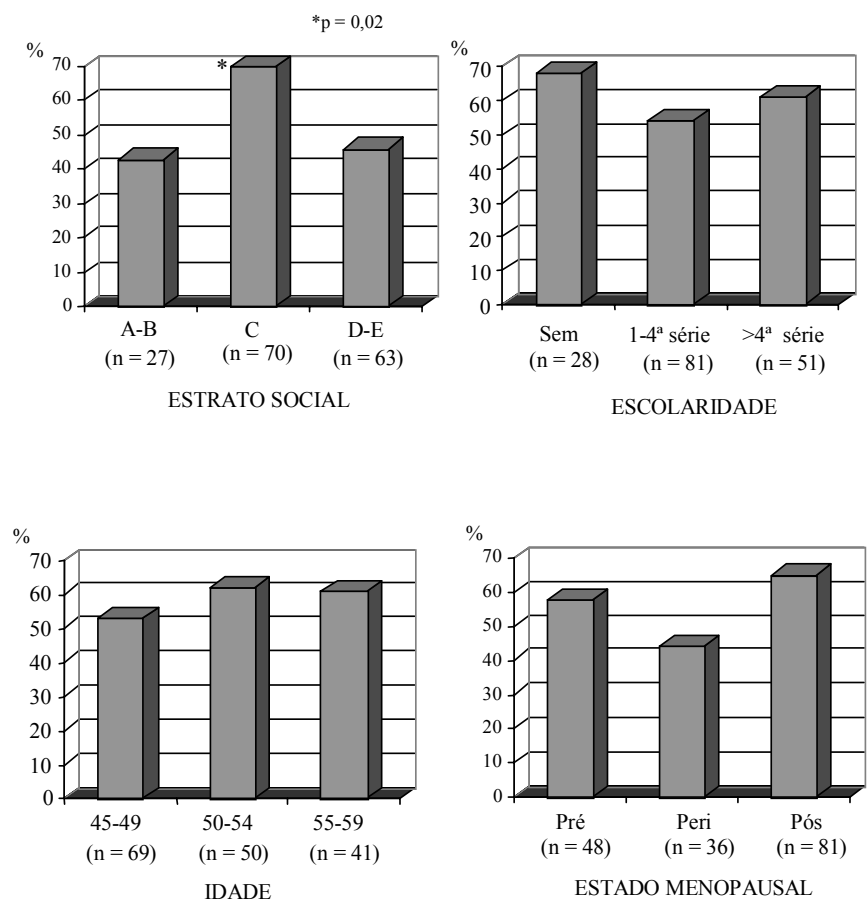

Figura 1 - Proporção de mulheres com queixa que consultaram médico segundo algumas características $(n=160)$.

Quase nove de cada dez mulheres que consultaram médico (87\%) por incontinência urinária referiram não ter sido receitado nenhum tratamento medicamentoso.

\section{Discussão}

A proporção de mulheres que referiu apresentar perda urinária aos esforços neste estudo de base populacional foi semelhante ao observado em estudo anterior, com pacientes que procuraram serviço médico de atenção à menopausa, em que a queixa de incontinência urinária de esforço foi observada em $30,7 \%$ das mulheres ${ }^{11}$. Estes resultados são também semelhantes aos encontrados por Chiarelli et al. ${ }^{12}$, que observaram prevalência de 36 e 35\% de perda urinária entre mulheres australianas de 45 a 50 anos e 70 a 75 anos, respectivamente. Outros autores têm descrito prevalências menores, $26 \%$ entre mulheres japonesas $^{13}$ e $16 \%$ em mulheres dinamarquesas com idade entre 40 e 60 anos $^{14}$. Diferenças étnicas e metodológicas podem ser responsáveis pela menor prevalência descrita nesses estudos.

A proporção de mulheres com incontinência e que consultou médico, na população deste estudo, foi idêntica à descrita por Stenberg et al. ${ }^{15}$ 
entre mulheres menopausadas (59 e 60\%). Apesar de $40 \%$ parecer proporção muito elevada de mulheres com sintomas, que não procuraram ajuda médica, esta porcentagem foi ainda maior na experiência de outros autores. Apenas 21,3\% das mulheres com incontinência urinária consultaram médico na experiência de Sanches Gonzalez et al. ${ }^{2}$ e somente $27 \%$ no estudo de Ushiroyama et al. ${ }^{13}$ com 3.940 mulheres japonesas menopausadas que consultaram em uma clínica ginecológica.

É interessante neste estudo a observação de que a porcentagem de mulheres que consultou médico, por perda urinária aos esforços, não foi dependente do nivel de instrução, estrato socioeconômico, idade e estado menopausal. Assim mesmo, o motivo pelo qual a grande maioria das mulheres não consultou em razão da perda urinária esteve associado à desvalorização do sintoma, ou seja, o incômodo da perda urinária não era suficiente para justificar uma consulta médica ou para ter prioridade sobre outras atividades que ocupavam seu tempo. Parece que é como se a perda urinária fosse uma ocorrência natural do avançar da idade e fizesse parte dos problemas que as mulheres têm que aceitar ao aproximar-se da velhice.

É ainda importante salientar que a maioria das mulheres com incontinência urinária não tem ciência de que seus sintomas podem ser melhorados e curados com tratamento médico adequa$\mathrm{do}^{13}$. A manifestação de muitas mulheres, $10 \%$ neste estudo, de não se sentirem com liberdade para falar com o médico sobre este problema, é indicativo da necessidade de mudar a atitude de alguns médicos que inibem suas pacientes de expressarem suas queixas.

Por outro lado, a incontinência urinária interfere diretamente com as atividades diárias dessas mulheres, de tal maneira que aquelas que sofrem desta moléstia apresentam indices mais baixos de qualidade de vida ${ }^{12}$. Conseqüentemente, autores como Ushiroyama et al. ${ }^{13}$ apontam a necessidade de proporcionar uma maior conscientização às mulheres pós-menopausadas quanto aos benefícios de procurar tratamento médico para incontinência urinária, a fim de melhorar a sua qualidade de vida.

Tudo isto justifica as recomendações de Sanches Gonzalez et al. ${ }^{2}$, que chamam a atenção para a necessidade de se identificarem as razões pelas quais as mulheres não consultam médico, e de se adotarem medidas para corrigir este problema. Da mesma forma, Fernandes et al. ${ }^{16}$ recomendam que os médicos informem e ofereçam recursos às suas pacientes, propiciando a cura ou a melhora dos seus sintomas urinários.
Estas recomendações contrastam com os resultados desta pesquisa. Observou-se aqui que, na grande maioria das vezes, o médico não receitou nenhum tipo de tratamento medicamentoso para o problema. Como atualmente são muitos os recursos para o tratamento conservador da incontinência urinária, desde comportamentais até drogas e fisioterapia, estes resultados parecem indicar uma falta de informação de alguns médicos que atenderam estas mulheres.

Desta forma, os resultados do presente estudo confirmam a necessidade de conscientizar as mulheres, bem como os médicos, quanto aos benefícios do tratamento para a incontinência urinária. Sugerem, ainda, que esta necessidade existe em todos os grupos socioeconômicos e em todas as idades, a partir dos 45 anos, pelo menos.

No momento em que é cada vez maior o número de pessoas de 45 anos ou mais que se mantêm em plena atividade e desejam usufruir de uma vida social sem restrições, torna-se imperativo contribuir para a melhoria da qualidade de vida das mulheres peri e pós-menopausadas. Portanto, faz-se necessária uma atitude positiva quanto ao diagnóstico e tratamento da incontinência urinária, de modo semelhante ao que ocorre com outras entidades clínicas como a osteoporose e os problemas cardiovasculares.

\section{SUMMARY}

Purpose: to evaluate the percentage of 45- to 60-year-old women complaining of urinary incontinence, who look for medical treatment, and the factors possibly associated with the decision of visiting the doctor and the reasons for not doing so.

Methods: a secondary analysis of a population-based survey on climacterium and menopause in women living in the city of Campinas, São Paulo state, was carried out through a descriptive, exploratory and cross-sectional population study. A total of 456 women between 45 and 60 years of age were selected through a sampling process. The age at menopause and its associated factors were evaluated, as well as the prevalence of climacteric symptoms, the use of medical care, self-perception of the health status, and the sociocultural, sociodemographic and socioeconomic characteristics. Urinary incontinence complaint and the search for medical help due to the presence of symptoms were explored. The data were collected through home interviews, using a structured and pretested questionnaire provided by the International Health Foundation/International Menopause Society and by the North American Menopause Society, and adapted by the authors. The statistical analysis was performed using Fisher's exact test.

Results: thirty-five percent of the interviewees reported 
constant or intermittent stress incontinence, although only $59 \%$ of the patients with the complaint sought medical help. Conclusion: the majority of the women presenting urinary incontinence do not complain to the doctor if they are not questioned objectively.

KEY WORDS: Urinary incontinence. Menopause. Medical care.

\section{Referências}

1. Hampel C, Wienhold D, Benken N, Eggersmann C, Thuroff JW. Prevalence and natural history of female incontinence. Eur Urol 1997; 32 Suppl 2:3-12.

2. Sanches Gonzalez R, Ruperez Cordero O, Delgado Nicolas MA, Mateo Fernandez R, Hernando Blasquez MA. The prevalence of urinary incontinence in a population over 60 treated in primary care. Aten Primaria 1999; 24:421-4.

3. Butler RN, Maby JI, Montella JM, Young GP. Urinary incontinence: keys to diagnosis of the older women. Geriatrics 1999; 54:22-6, 29-30.

4 - Von Mühlen DG, Kritz-Silverstein D, Barret-Connor E. A community based study of menopause symptoms and estrogen replacement in older women. Maturitas 1995; 22:71-8.

5. Instituto Brasileiro de Geografia e Estatística (IBGE). Recenseamento Geral da População, 1991. Rio de Janeiro: IBGE Editora, 1994.

6. Laurenti R, Mello-Jorge MLP, Lebrão ML, Gotlieb SLD. Estatística de saúde. $2^{\text {a }}$ ed. São Paulo: EPU; 1987. p.9-38.

7. Boulet MJ, Oddens BJ, Lehert P, Vemer HM, Visser A. Climacteric and menopause in seven Southeast Asian countries. Maturitas 1994; 19:157-76.
8. Utian WH, Schiff I. Nams-Gallup survey on women's knowledge, information sources, and attitudes to menopause and hormone replacement therapy. Menopause 1994; 1:39-48.

9. Almeida, Pergentino M. \& Wickerhauser, Hilda. O critério ABA/ABIPEME - em busca de uma solução. Um estudo e uma proposta submetidos à ABA - Associação Brasileira de Anunciantes e ABIPEME - Editora da Associação Brasileira dos Institutos de Pesquisa de Mercado. São Paulo, Abril a Junho, 1991.

10.Jaszmann L. Epidemiology of climacteric and postclimacteric complaints. In: Van Keep PA, Lauritzen C, editors. Ageing and estrogens. Front Hormone Res. Basel:Karger; 1973. p.22-4.

11.Guarisi T, Pinto-Neto AM, Paiva LHC, Pedro AO, Faúndes A. Fatores associados à prevalência de sintomas urinários em mulheres climatéricas. Rev Bras Ginecol Obstet 1997; 19:589-96.

12. Chiarelli $\mathrm{P}$, Brown $\mathrm{W}$, McElduff $\mathrm{P}$. Leaking urine: prevalence and associated factors in Australian women. Neurourol Urodyn 1999; 18:567-77.

13.Ushiroyama T, Ikeda A, Ueki M. Prevalence, incidence, and awareness in the treatment of menopausal urinary incontinence. Maturitas 1999; 33:127-32.

14. Moller LA, Lose G, Jorgensen T. The prevalence and bothersomeness of lower urinary tract symptoms in women 40-60 years of age. Acta Obstet Gynecol Scand 2000; 79:298-305.

15.Stenberg A, Helmer G, Holmberg L, Ulmsten U. Prevalence of postmenopausal symptoms in two age groups of elderly women in relation to oestrogen replacement therapy. Maturitas 1999; 33:229-37.

16.Fernandes CE, Morita MH, Ferreira JAS, Silva EP, Wehba S. Abordagem dos distúrbios do trato urinário na mulher pós-menopausada. Rev Paul Med 1990; 108:230-5.

\title{
RBBO
}

\author{
é uma publicação da FEBRASGO \\ e que aceita artigos provenientes de ginecologistas, \\ obstetras e de outras especialidades. \\ Portanto, publique!!! \\ Mande já seu artigo para RBGO
}

